\title{
UNIVERSITY OF RLOO \\ Management of Anticoagulation in Patients with Metastatic Castration- Resistant Prostate Cancer Receiving Abiraterone + Prednisone
}

\section{Samuel Dubinsky, BSc. PharmD Candidate. ${ }^{1}$ Alia Thawer, BScPhm. PharmD. RPh. ${ }^{2}$ Thomas R.J. McFarlane, BScPhm. PharmD. RPh. ${ }^{1,2}$ Urban Emmenegger, MD. ${ }^{2}$ Anne G. McLeod, MD. ${ }^{3}$}

University of Waterloo School of Pharmacy, Kitchener, Ontario. ${ }^{2}$ Odette Cancer Centre, Sunnybrook Health Sciences Centre, Toronto, Ontario. ${ }^{3}$ Depart of Medicine, Division of Medical Oncology and Hematology, Sunnybrook Health Sciences Centre, Toronto, Ontario.

\section{Background}

- Abiraterone acetate is an oral, irreversible inhibitor of CYP17A1, that has been shown to be an effective agent for the management of metastatic castration-resistant and castration-sensitive prostate cancer. ${ }^{1,2,3,4}$

- Abiraterone has been shown to be a strong inhibitor of CYP 2D6, CYP $2 \mathrm{C} 8$ and $C Y P 1 \mathrm{~A} 2$, and in vitro data suggests moderate inhibition of CYP 3A4, CYP 2C9, CYP 2C19. Additionally, a minimal inhibitory effect on Pglycoprotein ( $\mathrm{P}$-gp) has been shown. ${ }^{5,6}$

- A recent analysis at our institution revealed that $39 \%$ of men undergoing abiraterone+prednisone therapy used anticoagulants and/or antiaggregatory medications. ${ }^{7}$

- Cancer associated thrombosis is a leading cause of death in ambulatory cancer patients receiving chemotherapy, second only to progression of disease. ${ }^{8}$ As a result, it is important for clinicians to provide anticoagulation options that minimize the risk of bleeding complications.

\section{Objective}

- Review current literature on anticoagulation management in the oncology setting.

- Develop a management algorithm based on efficacy, safety and pharmacokinetic data to provide a point of care reference for clinicians managing patients on abiraterone + prednisone who require anticoagulation.

\section{Methods}

- A thorough review of available literature was preformed to further aid in developing the anticoagulation protocol for non-valvular atrial fibrillation and venous thromboembolism within this population

- After reviewing the literature, a clinical framework was designed by the authors to aid clinicians in the management of those patients receiving abiraterone + prednisone concurrently with an anticoagulant.

\section{Results}

Table 1.0. Pharmacokinetics of Anticoagulants

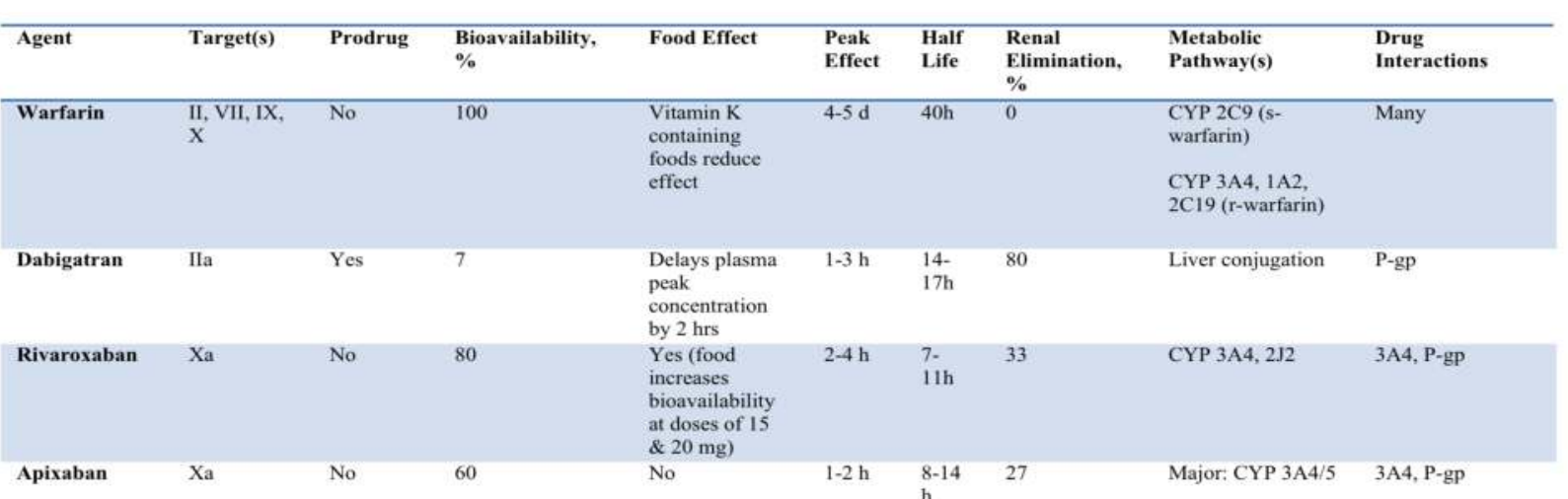
Minor: CYP 1A2,
$2 \mathrm{C}, 2 \mathrm{C} C, 2 \mathrm{C} 19$,

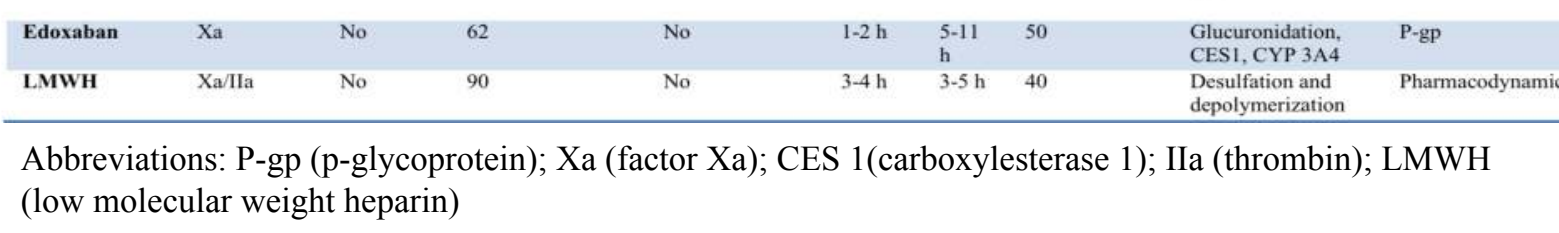
(low molecular weight heparin)

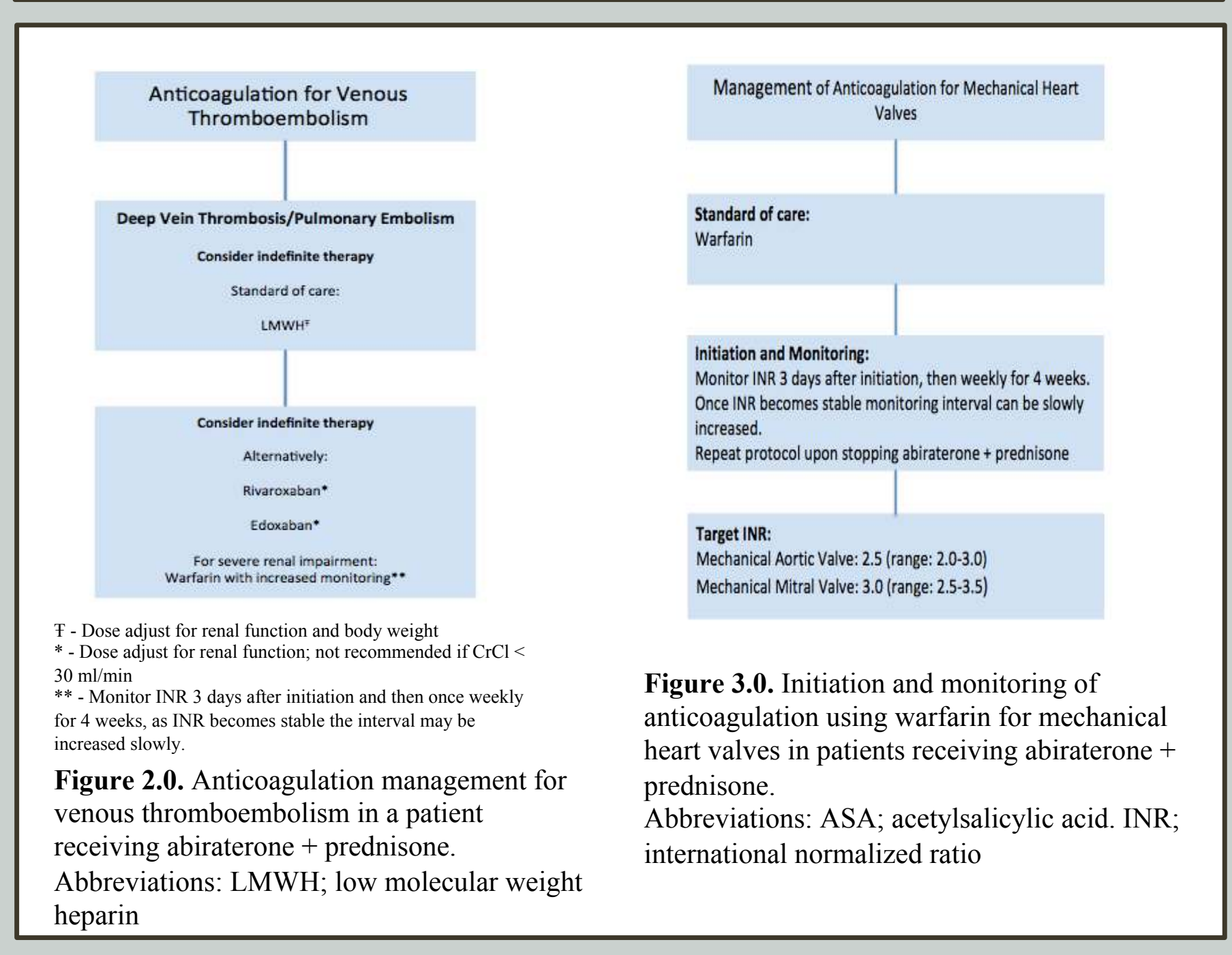

\section{Conclusion/Implications}

- Abiraterone + prednisone therapy has become a mainstay of the management of advanced prostate cancer, and is often used over prolonged periods of time.

- We have summarized herein a framework of how to safely use abiraterone + prednisone in men with prostate cancer on anticoagulants, applying best current knowledge.

- Evidence available to date suggests that patients with an indication for anticoagulation such as atrial fibrillation, VTE and mechanical heart valves can be treated safely with abiraterone + prednisone with appropriate monitoring.

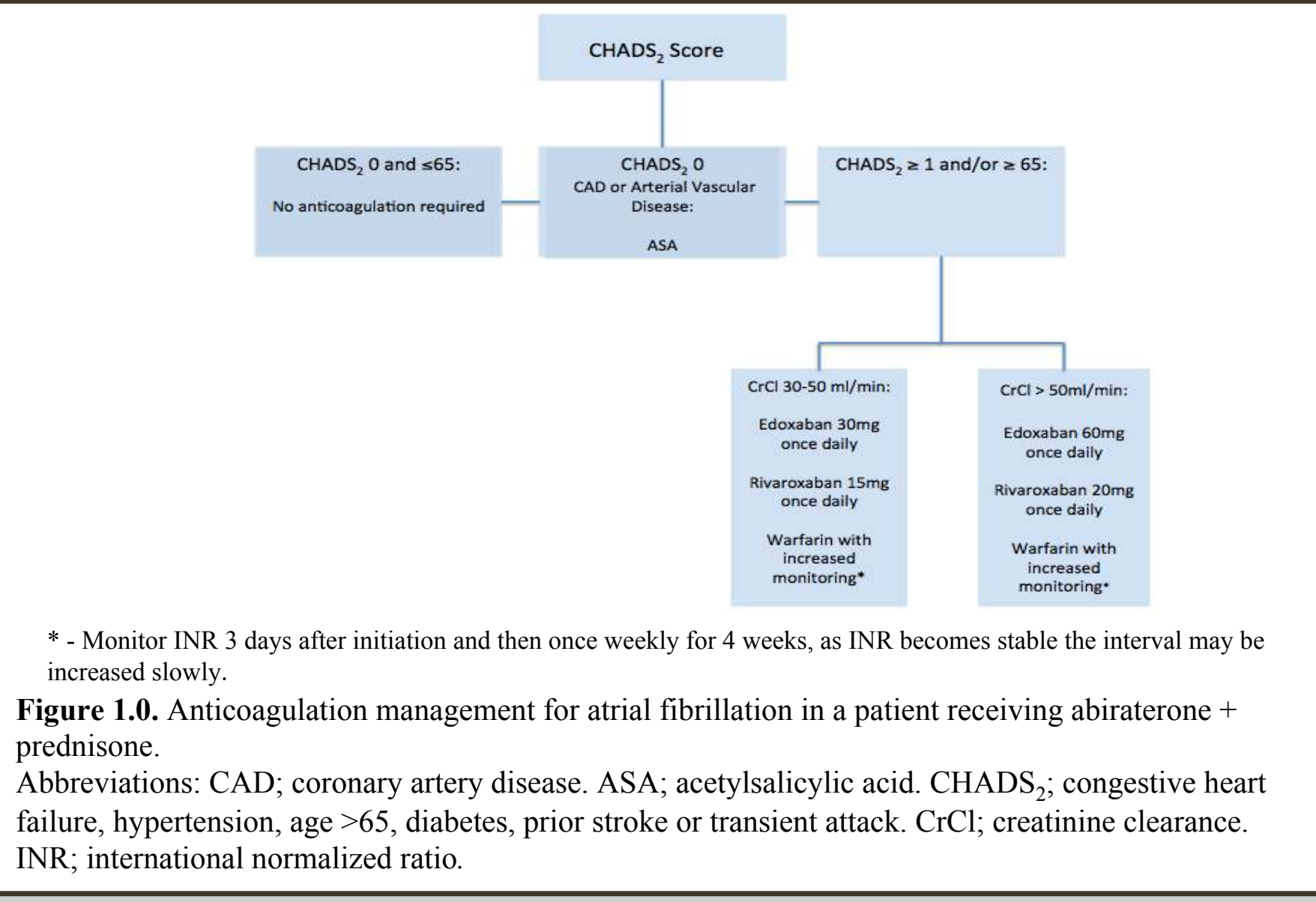

Table 2.0. Recommended management strategies for anticoagulation in patients receiving abiraterone prednisone.

Atrial Fibrillation: Therapy:

$\mathrm{CHADS}_{2}$ Score 0 and age $\leq 65$

$\mathrm{CHADS}_{2}$ Score 0 and CAD

$\mathrm{CHADS}_{2}$ Score $\geq 1$ and/or age $\geq 65$

No anticoagulation

ASA

Anticoagulation with Edoxaban, Rivaroxaban or Warfarin

\section{Deep Vein Thrombosis/ Pulmonary Embolism}

LMWH is standard of care. Rivaroxaban or Edoxaban as alternative.

Mechanical Heart Valve

Warfarin* is the only acceptable anticoagulant.

* - Close monitoring of INR

Abbreviations: ASA, acetylsalicylic acid; CAD; coronary artery disease. $\mathrm{CHADS}_{2}$, Congestive heart failure, hypertension, age, diabetes, prior stroke or transient attack; LMWH, low-molecular weight heparin.

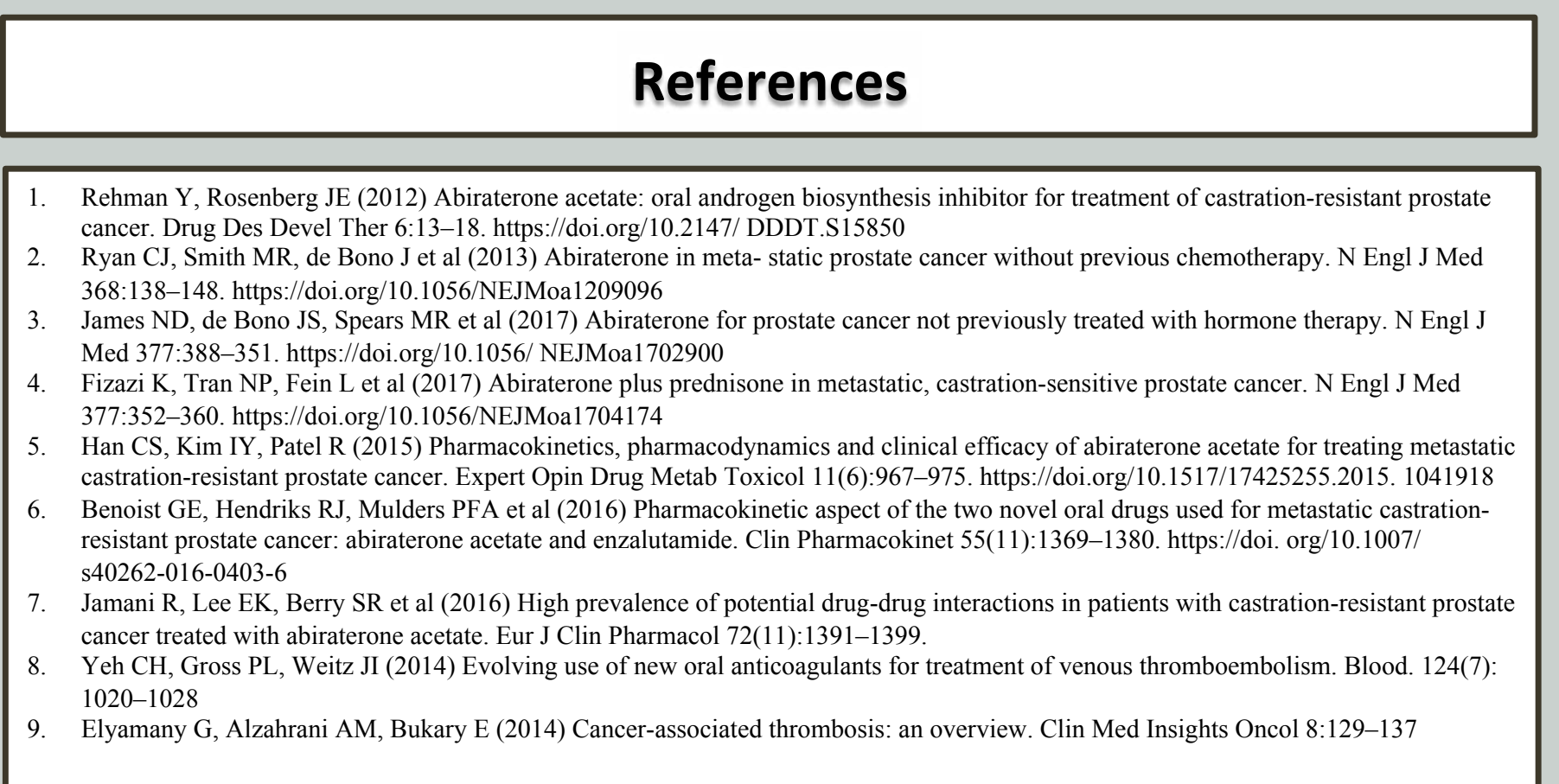

